\title{
Isolated oxycephaly
}

INSERM

\section{Source}

INSERM. (1999). Orphanet: an online rare disease and orphan drug data base. Isolated oxycephaly. ORPHA:63440

Isolated oxycephaly is a late-appearing form of nonsyndromic craniosynostosis characterized by premature fusion of both the coronal and sagittal sutures, and, in some cases, of the lambdoid sutures. Compensatory growth in the region of the anterior fontanel results in a pointed or cone-shaped skull. 